\title{
Volatilidad estocástica del tipo de cambio peso-dólar: el régimen flotante en México
}

\author{
Francisco López Herrera \\ Domingo Rodríguez Benavides \\ Francisco Ortiz Arango*
}

\section{TIPO DE CAMBIO Y RIESGO CAMBIARIO}

La apertura de los mercados ha producido un incremento sostenido tanto en las relaciones de intercambio comercial como en la circulación de los capitales destinados a la inversión internacional de portafolios. Como se sabe, en primera instancia, las fluctuaciones en el tipo de cambio dificultan la toma de decisiones relacionadas con el comercio y la inversión internacional de portafolios pues crean riesgo para las empresas e individuos en el sector privado. Por otra parte, también puede verse que estas fluctuaciones cambiarias son percibidas por algunos inversionistas como magníficas oportunidades de inversión como lo sugieren la intensa actividad y los cuantiosos volúmenes de las negociaciones que se llevan a cabo en el mercado internacional

\footnotetext{
Manuscrito recibido en diciembre de 2010; aceptado en marzo de 2011.

* Facultad de Contaduría y Administración de la Universidad Nacional Autónoma de México (UNAM), <francisco_lopez_herrera@yahoo.com.mx>; División de Ciencias Sociales y Administración de la Universidad Autónoma Metropolitana, Unidad Azcapotzalco (UAM-Az), <domr@economia.unam.mx>, y Escuela de Ciencias Económicas y Empresariales de la Universidad Panamericana, <fortizar@up. edu.mx $>$, respectivamente. Los autores de este trabajo agradecen los comentarios y sugerencias que hicieron amablemente dos dictaminadores anónimos para mejorarlo, aceptando la responsabilidad por cualquier error que aún persista.
} 
de divisas. La volatilidad en el tipo de cambio, junto con la volatilidad de los precios de otros activos financieros, ha fomentado el desarrollo de nuevos mercados e instrumentos que pueden en todo caso reducir o incrementar la exposición a las condiciones de la volatilidad cambiaria. Para los tipos de cambio en particular se puede señalar la ruptura del sistema internacional de paridades cambiarias como los antecedentes de la situación de flotación generalizada de las principales divisas del mundo.

Entre los acontecimientos que dispararon la ruptura del sistema cambiario internacional establecido desde los acuerdos de Bretton Woods a finales de la Segunda Guerra Mundial, se puede mencionar la devaluación de la libra esterlina en noviembre de 1967, a la cual le siguió una erupción de episodios de presión sobre el sistema internacional de paridades cambiarias: la revaluación del marco alemán en 1969, la flotación del dólar canadiense en 1970; temporalmente flotaron el marco alemán y el florín holandés en 1971, seguidas por el hecho de que empezaron a flotar la mayoría de las principales divisas desde el 15 de agosto de 1971 hasta el acuerdo Smithsoniano en diciembre de ese mismo año. Todo ese conjunto de episodios propició que el International Monetary Market (IMM) del Chicago Mercantile Exchange (CME) introdujera el comercio de futuros del tipo de cambio en mayo de 1972. Una vez que la libra británica empezó a flotar en junio de 1972 le siguió una flotación generalizada de otras divisas importantes a principios de 1973. Como consecuencia directa de los efectos de la ruptura del sistema de paridades fijas, las necesidades de cobertura se incrementaron y con ellas, obviamente, crecieron las perspectivas de desarrollo de los mercados de productos diseñados específicamente para fines de cobertura de la volatilidad de los tipos de cambio.

Se ha argumentado que los sistemas de flotación cambiaria pueden ser más riesgosos que los sistemas de paridades fijas, es decir, se espera que la volatilidad de los tipos de cambios cuando hay libre flotación sea mayor pues se permite que la paridad esté modificándose de manera prácticamente constante. $\mathrm{Al}$ respecto es conveniente destacar que el estudio de Clark et al. (2004) muestra que de 1970 a 1990, en promedio, la volatilidad de los tipos de cambio fijos ha sido de manera aproximada la misma que con los tipos de 
cambio flotantes. Sin embargo, no deben interpretarse los hallazgos de ese estudio como evidencia de que el riesgo cambiario es trivial o que se puede ignorar. En última instancia, Clark et al. (2004) sólo dan cuenta de que la volatilidad cambiaria no afecta más a los flujos comerciales cuando el tipo de cambio es flexible, siendo sus efectos equiparables independientemente de que el sistema cambiario sea fijo o flexible. Naturalmente, esto no implica que la volatilidad sea irrelevante para el comercio internacional, pues no se ha zanjado la discusión con respecto a si la volatilidad cambiaria es un obstáculo para los intercambios comerciales dados los costos de cobertura. Por ejemplo, entre quienes muestran efectos negativos de la volatilidad del tipo de cambio se encuentran Clark (1973), Ethier (1973), Hooper y Kohlhagen (1978), Cushman (1988) y Wolf (1995). Franke (1991), De Grauwe (1988) y Giovannini (1988) muestran que la volatilidad cambiaria no sólo no obstaculiza los flujos comerciales sino que incluso puede estimularlos porque, por ejemplo sostiene De Grauwe (1988), puede existir un cierto nivel de aversión al riesgo de un productor en el cual un incremento del riesgo cambiario puede elevar su utilidad marginal esperada del ingreso proveniente de la exportación, induciéndolo así a tratar de incrementar su oferta de exportación. La investigación empírica no es concluyente, incluso en un estudio reciente Baum y Caglayan (2009) encuentran una relación débil entre la volatilidad cambiaria y los flujos comerciales de 22 países durante el periodo 1980-2006, entre la muestra de países se encuentra México. Sin embargo, a juzgar por el amplio uso de instrumentos para cobertura cambiaria por parte de empresas involucradas en operaciones internacionales, el riesgo derivado de la volatilidad cambiaria sigue siendo un elemento de suma importancia a considerar en las relaciones de intercambio comercial en el ámbito internacional.

La inversión de portafolios diversificados internacionalmente ha venido incrementándose de manera más o menos sostenida en la medida en que se han facilitado ese tipo de transacciones mediante la desregulación de los mercados financieros alrededor del mundo. Se puede suponer que el riesgo cambiario que enfrentan los inversionistas internacionales puede constituirse en un obstáculo de importancia para frenar o, al menos, desalentar los flujos 
de la inversión de portafolios. Es sabido que los mercados y productos para negociar coberturas cambiarias permiten: i) administrar el riesgo de manera ágil y con un costo relativamente bajo sin necesidad de recurrir a estrategias dinámicas de inversión que incrementan notoriamente los costos derivados de las transacciones requeridas por el rebalanceo de los portafolios; ii) que los agentes puedan participar en los mercados internacionales de manera más flexible ya que con ellos se puede invertir a partir de la expectativa de mercado con que cuenten; iii) invertir incluso sin tener una idea totalmente clara sobre los movimientos de los precios; iv) contar con un grado adecuado de protección contra movimientos adversos del mercado y v) aprovechar con mayor facilidad las discrepancias de precio que pueden presentarse entre productos similares y muchas otras estrategias que pueden basarse en arbitraje. En última instancia, a pesar del enorme desarrollo de los mercados e instrumentos de cobertura cambiaria, de cualquier forma es claro que la volatilidad cambiaria incrementa el riesgo de pérdidas relacionadas con el programa de inversión o, al menos, puede incrementar los costos de protección contra los riesgos derivados de las fluctuaciones cambiarias.

En resumen, el riesgo cambiario sigue constituyéndose como importante para toda actividad económica que implique intercambios internacionales, sean de activos físicos o de activos financieros. Adicionalmente es importante considerar que se ha observado que el comportamiento de las variables económicas puede mostrar rupturas drásticas como una de las consecuencias del surgimiento y desarrollo de crisis financieras, como lo evidencian las crisis que México ha enfrentado reiteradamente desde la segunda mitad de los años setenta a épocas más recientes. Por ejemplo, Rodríguez y Venegas-Martínez (2011) muestran evidencia de rupturas en el producto interno bruto (PIB) mexicano ocurridas, entre otros años, en 1980, 1988 y 2007, fácilmente identificables como consecuencia de periodos de crisis. También se ha observado que en las series de datos financieros es muy común encontrar rupturas, sean éstas derivadas de los efectos de su sensibilidad al comportamiento de las variables económicas o, en muchas ocasiones, de eventos propios de los mercados financieros. Como ejemplo, puede citarse el trabajo de López-Herrera y Ortiz (2011) en el que muestran 
evidencia de que las primas de riesgo de los mercados del área del Tratado de Libre Comercio de América del Norte (TLCAN) han sufrido los efectos del comportamiento de variables económicas locales e internacionales así como de crisis en los mercados financieros locales y foráneos. Debido a la interrelación entre variables económicas y financieras, la inestabilidad cambiaria puede tener efectos negativos en la actividad económica agregada, afectando por lo tanto no sólo a empresas e inversionistas involucrados en actividades internacionales, sino también a empresas e inversionistas cuyo enfoque sólo es local y, en última instancia, los efectos de la inestabilidad del tipo de cambio pueden alcanzar a los consumidores nacionales.

De manera general, el análisis de la volatilidad cambiaria sigue siendo un tema de importancia en la agenda de investigación económica y financiera, pues no sólo resulta ser de utilidad para la selección de inversiones y la administración de riesgos mediante el diseño de coberturas adecuadas, sino también lo es para el diseño de políticas congruentes con la estabilidad cambiaria y que propicien el crecimiento de la economía. En lo particular, el estudio de la volatilidad en la paridad cambiaria del peso mexicano respecto al dólar es de gran importancia dadas nuestras tradicionales relaciones comerciales con Estados Unidos, las que se han estrechado más aún a partir de la entrada en vigor del Tratado de Libre Comercio de América del Norte profundizando así la dependencia del desempeño de la economía mexicana respecto del desempeño de la economía de ese país.

En algunos estudios sobre la volatilidad cambiaria se han utilizado modelos generalizados de heterocedasticidad condicional autorregresiva (GARCH, por sus siglas en inglés) para analizarla. Por ejemplo, Sengupta y Sfeir (1996) estudian la volatilidad cambiaria del dólar estadounidense respecto de la libra esterlina, el yen, el marco alemán y el franco francés; Baillie et al. (1996) analizan la volatilidad del tipo de cambio del marco alemán respecto del dólar; Tse (1998) estudia la volatilidad del tipo de cambio yen-dólar. Trabajos más recientes también han seguido esta línea de modelado: Longmore y Robinson (2004) analizan el comportamiento del dólar de Jamaica y el dólar de Estados Unidos. Chowdhury y Sarno (2004) y McMillan y Speight (2006) estudian la dinámica de los tipos de cambio con datos intradía. Sandoval (2006) analiza 
la volatilidad cambiaria de las monedas de algunos países con mercados financieros emergentes. ${ }^{1}$ Bauwens y Sucarrat (2008) estudian la volatilidad de la corona noruega; Olowe (2009) analiza la volatilidad cambiaria de la moneda nigeriana respecto del dólar de Estados Unidos; Siddiqui (2009) analiza la volatilidad de la paridad cambiaria de la rupia paquistaní en relación con las monedas de Canadá, Estados Unidos, Japón, Reino Unido y la Unión Monetaria Europea; Mohnot (2011) estudia la capacidad que tienen los modelos GARCH para predecir la volatilidad cambiaria respecto al dólar estadounidense de las monedas de 13 países (entre los cuales se incluye a México). Otros estudiosos han optado por analizar la volatilidad cambiaria utilizando modelos de volatilidad estocástica. Taylor (1994) estudia el tipo de cambio diario del marco alemán respecto del dólar aplicando varios modelos de volatilidad estocástica. Mahieu y Schotman (1998) analizan la volatilidad de los tipos de cambio bilaterales de las monedas de Estados Unidos, Japón, Alemania y el Reino Unido. Taylor (1999) utiliza un modelo de volatilidad estocástica para estudiar la volatilidad del tipo de cambio de la libra esterlina respecto del dólar estadounidense. Arranz e Iglesias (2005) analizan el comportamiento del tipo de cambio peso-dólar, comparándolo con el de los tipos de cambio de Venezuela, la República Sudafricana, Noruega y del Reino Unido, utilizando el modelo de volatilidad estocástica propuesto por Taylor (1986).

Desde nuestra perspectiva consideramos que en un entorno de volatilidad es importante no sólo contar con estimaciones de la misma y de la posibilidad de pronosticar la volatilidad del periodo siguiente, sino que también es de gran utilidad para los tomadores de decisiones contar con información respecto a las probabilidades que tienen de observar baja o alta volatilidad, la probable duración de cada uno de los estados en que se puede encontrar la volatilidad, es decir, cuánto se espera que dure un periodo de alta (baja) volatilidad y cuál es la probabilidad de que se pase a un estado de alta volatilidad cuando el tipo de cambio se encuentra en el estado de baja volatilidad o viceversa. Naturalmente, se puede observar la paridad cambiaria mediante

\footnotetext{
${ }^{1}$ Brasil, Colombia, Corea del Sur, Chile, India, México y Tailandia.
} 
datos del mercado de cambios y, gracias ello, también se puede inferir de manera más o menos inmediata la tasa de depreciación/apreciación de una moneda frente a otra; sin embargo, las probabilidades antes señaladas no son observables directamente y, por tanto, deben inferirse a partir de la información disponible para los agentes económicos. Por tal motivo, este trabajo se enfoca en el análisis de la volatilidad del tipo de cambio peso-dólar, con el objetivo de indagar respecto a la pertinencia de modelar la volatilidad cambiaria peso-dólar mediante un modelo de volatilidad estocástica que considere los cambios de estado o régimen de la volatilidad cambiaria para dar respuesta a los cuestionamientos que se han enunciado líneas antes.

La estructura del presente trabajo es la que se enuncia a continuación. La sección siguiente presenta el modelo de volatilidad estocástica basado en una cadena de Markov con dos estados que se emplean en nuestro análisis de la volatilidad del tipo de cambio peso-dólar y cuyos resultados se dan en la tercera sección en la cual también se presenta, a manera de antecedentes, una breve descripción del comportamiento observado de la paridad cambiaria de la moneda mexicana respecto al dólar a partir de enero de 1995. Finalmente, la cuarta sección contiene las conclusiones del análisis de la volatilidad cambiaria peso-dólar.

\section{Modelo de volatilidad CAMbiaRia eSTOCÁstica}

Se atribuye a Prasad et al.(1974) el haber presentado el primer modelo financiero en que se utilizó una cadena de Markov. Uno de los trabajos más conocidos que se basa en ese enfoque es el de Hamilton (1989) en el cual extiende la regresión de cambios markovianos de Goldfeld y Quandt (1973), presentando el uso de procesos discretos de Markov para modelar los cambios ocasionales y discretos en la tasa media de crecimiento de una serie no estacionaria, mostrando la aplicación de su técnica al análisis del producto nacional bruto (PNB) real de Estados Unidos después de la Segunda Guerra Mundial. El modelo de Hamilton (1989) le permitió tomar en cuenta los comportamientos diferenciados que observó Neftci (1984) en el comportamiento de variables económicas relacionadas con el empleo, 
diferenciados de acuerdo con la fase del ciclo económico. Además, con su enfoque Hamilton fue capaz de reproducir adecuadamente los puntos de cambio de fase del ciclo económico de acuerdo con la imputación de fechas elaborada por el National Bureau of Economic Research (NBER). En uno de sus trabajos recientes, Hamilton (2005) efectúa una revisión del comportamiento del desempleo en Estados Unidos y de la tasa de interés y ofrece evidencia de que en ambos casos se logra un mejor ajuste en los modelos cuando se considera que existen cambios de régimen.

Otras aplicaciones relevantes de los modelos basados en cadenas de Markov son la de Hamilton (1988) a tasas de interés y la de Engel y Hamilton (1990) a los tipos de cambio. Otros trabajos que se pueden citar también como aplicaciones interesantes de las cadenas de Markov son el de Taylor (1999) sobre el tipo de cambio libra-dólar; el de Masson (2000) en el cual, basándose en este enfoque de modelado, se analizan las transiciones entre regímenes cambiarios en un amplio estudio que incluyó a todos los países para los cuales el Fondo Monetario Internacional (FMI) tenía datos para esa fecha, y el trabajo de Jeanne y Masson (2000) en el que muestran que los modelos basados en cambios de régimen markovianos pueden ser de gran utilidad para analizar empíricamente las crisis cambiarias con base en modelos de equilibrio múltiples en los que los inversionistas saben que hay posibilidades de que la economía dé saltos entre diferentes estados asociados con diferentes niveles de devaluación del tipo de cambio e incorporan, racionalmente, esas expectativas a sus decisiones pudiendo dar como resultado un proceso de profecías autocumplidas pues los efectos de esas decisiones pueden verse reflejados a su vez como un incremento en la volatilidad de los mercados financieros. Jeanne y Masson (2000) ilustran la aplicación de su enfoque de modelado mediante el análisis del comportamiento que se observó en el franco francés entre 1987 y 1993, periodo en que la moneda francesa enfrentó fuertes presiones cambiarias.

En el caso mexicano es de comentar el trabajo llevado a cabo por Ramírez y Sandoval-Saveedra (2002) quienes extienden, a su vez, el trabajo de McQueen y Thorley (1991) y con base en ese enfoque detectan elementos pronosticables en un portafolio formado por acciones que cotizan en la Bolsa Mexicana de 
Valores, resultados que son de interés a la luz de las predicciones efectuadas por la hipótesis de los mercados eficientes en la cual se basa el grueso de la teoría financiera. Sobre este punto, Perrotini y Rodríguez (2007) evalúan la hipótesis de los mercados eficientes para las coberturas cambiarias, tanto de los futuros del peso mexicano como las del mercado no estandarizado. Ellos argumentan que las pruebas de cointegración entre el tipo de cambio spot y la tasa forward reflejan la existencia de un arbitraje de acuerdo con la paridad cubierta de tasas de interés y no, como es generalmente aceptado, la eficiencia en el mercado, de acuerdo con lo que plantean Kellard et al. (2001). De esta manera, se considera que hay información adicional en las tasas de interés que explica las ineficiencias cambiarias.

Por otra parte, es ampliamente conocido que en las distribuciones de los rendimientos de activos financieros se observan grandes picos, colas pesadas y exceso de curtosis en comparación con lo que se esperaría en la distribución normal; además, se reconoce como hecho indiscutible que la volatilidad varía en el tiempo como lo mostraron de manera temprana Mandelbrot (1963) y Fama (1965), hecho reconocido posteriormente por Black y Scholes (1972). Como también se sabe, para enfrentar estos hechos estilizados de los rendimientos de los activos financieros surgieron los modelos de volatilidad variante en el tiempo o volatilidad condicional como los modelos de heterocedasticidad condicional autorregresiva (ARCH, por sus siglas en inglés) o los de volatilidad estocástica cuya versión estándar es la de Taylor (1982). Una representación bastante general del proceso de los rendimientos, por ello sumamente popular en la literatura de investigación financiera, misma que consiste en representar el rendimiento del periodo $t$ como lo propone Taylor (2005): ${ }^{2}$

$$
\begin{gathered}
r_{t}=\mu+\sigma_{t} u_{t} \\
u_{t} \sim \operatorname{iid} N(0,1)
\end{gathered}
$$

\footnotetext{
${ }^{2}$ Es conveniente decir que Lindgren (1978) y Baum et al. (1980) ofrecen por primera vez el análisis de una representación bastante similar a la que aquí se describe.
} 
Una de las explicaciones que se han dado respecto a la presencia de los hechos estilizados, previamente mencionados, es que la distribución de los rendimientos es una mezcla de distribuciones normales con varianzas diferentes. Partiendo de este planteamiento, Hamilton (1988) propone la construcción de un proceso estocástico relativamente simple para modelar la volatilidad de los rendimientos, basándose en el supuesto de que el nivel de la volatilidad varía en el tiempo bajo la conducción de una cadena de Markov con una distribución discreta de probabilidades que tiene únicamente dos estados:

$$
\begin{gathered}
\sigma_{t}=\left\{\begin{array}{l}
\sigma_{\text {baja }} \text { con probabilidad } p \\
\sigma_{\text {alta }} \text { con probabilidad } 1-p
\end{array}\right. \\
\sigma_{t}>0, \sigma_{\text {baja }}<\sigma_{\text {alta }}
\end{gathered}
$$

En principio el considerar únicamente dos estados o regímenes para la volatilidad es un enfoque simplificador pues sólo se permite que la volatilidad se encuentre en un nivel alto o un nivel bajo, situación que resulta conveniente pues evidentemente se facilita la estimación de los parámetros del modelo de volatilidad estocástica cuyos detalles se presentan en los párrafos siguientes de esta sección. Por otra parte, más allá de un punto de vista pragmático, se puede argumentar también que las medidas que la autoridad monetaria ha instrumentado para la estabilidad cambiaria tienden a buscar que la volatilidad del tipo de cambio peso-dólar sea baja, estado o régimen de volatilidad que puede considerarse afín con las expectativas de que no habrá movimientos bruscos en la paridad cambiaria. Es decir, cualquier otro estado indicativo de una mayor volatilidad, sea ésta media o alta, implica la existencia de expectativas de inestabilidad del comportamiento cambiario a pesar de las medidas tomadas por el Banco Central para preservar la estabilidad del tipo de cambio. Finalmente, basándonos en el comportamiento de los movimientos del tipo de cambio peso-dólar, se brindará en la sección siguiente una justificación empírica de la decisión para modelar la volatilidad cambiaria peso-dólar con sólo dos estados. 
Debido a que se supone que $\left\{\sigma_{t}\right\}$ es un proceso de Markov, la probabilidad de que el rendimiento del activo pase al régimen de alta volatilidad cuando se encuentra en el régimen que corresponde a la volatilidad baja depende únicamente del último estado en que se encontraba el proceso, es decir, la probabilidad de transición del estado de baja volatilidad al de alta volatilidad está dada por:

$$
p_{b a}=P\left(\sigma_{t}=\sigma_{\text {alta }} \mid \sigma_{t-1}=\sigma_{\text {baja }}\right)
$$

y la probabilidad de transición del régimen de volatilidad alta al régimen de baja volatilidad por:

$$
p_{a b}=P\left(\sigma_{t}=\sigma_{\text {baja }} \mid \sigma_{t-1}=\sigma_{\text {alta }}\right)
$$

Entonces los rendimientos se distribuyen $N\left(\mu, \sigma_{b a j a}^{2}\right)$ cuando la volatilidad se encuentra en el régimen de baja volatilidad y $N\left(\mu, \sigma_{\text {alta }}^{2}\right)$ cuando la volatilidad se encuentra en el régimen en el cual predomina la volatilidad alta. Basándose en el supuesto de que el proceso $\left\{\sigma_{t}\right\}$ es estacionario estrictamente y también independiente estocásticamente de $\left\{u_{t}\right\}$, es posible derivar fórmulas para los momentos de $r_{t}-\mu=\sigma_{t} u_{i}$; como lo señala Taylor (2005). Así, de acuerdo con [1] y [2], $E\left(r_{t}\right)=\mu$ y $\operatorname{var}\left(r_{t}\right)=E\left[\sigma_{t}^{2}\right]$. La varianza no condicional de los rendimientos es:

$$
\sigma^{2}=\operatorname{var}\left(r_{t}\right)=E\left(\sigma_{t}^{2}\right)=p \sigma_{\text {baja }}^{2}+(1-p) \sigma_{\text {alta }}^{2}
$$

También se tiene como resultado que la densidad no condicional de los rendimientos es una mezcla de densidades normales:

$$
f\left(r_{t}\right)=p \psi\left(r_{t} \mid \mu, \sigma_{b a j a}^{2}\right)+(1-p) \psi\left(r_{t} \mid \mu, \sigma_{\text {alta }}^{2}\right)
$$

En la ecuación $[6], \psi(\bullet)$ representa la función de densidad normal. También con base en los supuestos respecto a $\left\{\sigma_{t}\right\}$ y $\left\{u_{t}\right\}$, anteriormente mencionados, tenemos que: 


$$
\sigma_{t}^{2}=\sigma^{2}+\left(1-p_{b a}-p_{a b}\right)\left(\sigma_{t-1}^{2}-\sigma^{2}\right)+\xi_{t}
$$

Según Pagan (1996), $\xi_{t}$ es un ruido blanco de acuerdo con el teorema de descomposición de Wold (1938) para un proceso estacionario, incluso aunque $\xi_{t}$ no sea $i i d$. De acuerdo con [7] $\left\{\sigma_{t}^{2}\right\}$ es un proceso $A R(1)$ con un parámetro autorregresivo:

$$
\phi=1-p_{b a}-p_{a b}
$$

el cual mide la persistencia de la volatilidad y cuyo valor empírico se espera que por lo común sea cercano a 1 , implicando que las probabilidades de transición o de cambio de estado (régimen), $p_{b a}$ y $p_{a b}$, deben ser pequeñas. Además, una persistencia alta en volatilidad implica que los choques en ella no se disipan rápidamente.

La información que proporcionan los rendimientos no es suficiente para identificar el estado de la volatilidad en algún momento del tiempo, por lo que se tiene que recurrir a las probabilidades condicionales del régimen o estado en que se encuentra la volatilidad, condicionado el estado actual por el comportamiento de los rendimientos previos, es decir:

$$
p_{t}=P\left(\sigma_{t}=\sigma_{b} \mid \Omega_{t-1}\right) \text { y } q_{t}=P\left(\sigma_{t}=\sigma_{a} \mid \Omega_{t-1}\right)
$$

$\Omega_{t-1}$ es el conjunto de información constituido por el historial de los rendimientos hasta el periodo $t-1$. Suponiendo que ya se han obtenido $p_{t-1} \mathrm{y}$ $q_{t-1}$ a partir de $\Omega_{t-2}$, estas probabilidades a priori se pueden revisar con base en el teorema de Bayes una vez que $r_{t-1}$ está disponible para obtener las probabilidades revisadas o posteriores:

$$
\begin{gathered}
p_{t-1}^{*}=P\left(\sigma_{t-1}=\sigma_{b} \mid \Omega_{t-1}\right)=\frac{p_{t-1} \psi\left(r_{t-1} \mid \mu, \sigma_{b}^{2}\right)}{p_{t-1} \psi\left(r_{t-1} \mid \mu, \sigma_{b}^{2}\right)+q_{t-1} \psi\left(r_{t-1} \mid \mu, \sigma_{a}^{2}\right)} \\
q_{t-1}^{*}=1-p_{t-1}^{*}
\end{gathered}
$$


A partir de las probabilidades de transición de la cadena de Markov, se obtienen también las probabilidades previas:

$$
p_{t}=p_{t-1}^{*}\left(1-p_{b a}\right)+q_{t-1}^{*} p_{a b}
$$

Al combinar [10] y [11] se obtienen las probabilidades condicionales:

$$
p_{t}=\frac{p_{t-1} \psi\left(r_{t-1} \mid \mu, \sigma_{b}^{2}\right)\left(1-p_{b a}\right)+q_{t-1} \psi\left(r_{t-1} \mid \mu, \sigma_{a}^{2}\right) p_{a b}}{p_{t-1} \psi\left(r_{t-1} \mid \mu, \sigma_{b}^{2}\right)+q_{t-1} \psi\left(r_{t-1} \mid \mu, \sigma_{a}^{2}\right)}
$$

Esta es la fórmula recursiva que comienza con $p_{1}=p .^{3}$

La densidad condicional de $r_{t}$, dados los rendimientos previos $\Omega_{t-1}$, también es una mezcla de distribuciones normales:

$$
f\left(r_{t} \mid \Omega_{t-1}\right)=p_{t} \psi\left(r_{t} \mid \mu, \sigma_{b}^{2}\right)+\left(1-p_{t}\right) \psi\left(r_{t} \mid \mu, \sigma_{a}^{2}\right)
$$

Finalmente, la varianza condicional está dada por el proceso:

$$
h_{t}=p_{t} \sigma_{b}^{2}+\left(1-p_{t}\right) \sigma_{a}^{2}
$$

En conclusión, las estimaciones de las probabilidades condicionadas, así como de las probabilidades de transición y las varianzas condicionadas se pueden obtener como subproductos de la estimación de los parámetros de interés $\mu, \sigma_{b a j a}, \sigma_{\text {alta }}, p$ y $\phi$. Esta estimación puede llevarse a cabo al optimizar el logaritmo de la función de verosimilitud, es decir, la suma de los logaritmos de las contribuciones individuales que para cada observación hace la ecuación [13].

\footnotetext{
${ }^{3}$ Siendo la probabilidad de volatilidad baja, $p$, uno de los parámetros del modelo cuyo valor se debe estimar, la estimación se obtiene como el valor que inicia la serie de probabilidades condicionadas $p_{t}$ y que es un valor óptimo en el sentido que al mismo tiempo permite maximizar la suma de los logaritmos de la función de verosimilitud dada por la ecuación [13].
} 


\section{El TIPO DE CAMBIO PESO-DÓlaR EN EL RÉGIMEN DE LIBRE FLOTACIÓN}

Después de experimentar diferentes sistemas cambiarios para mantener una paridad fija y que los fracasos para sostenerla produjeran sucesivas devaluaciones, a partir de enero de 1991 la paridad cambiaria del peso mexicano con el dólar estadounidense se fijó mediante un sistema de bandas de flotación cuya amplitud se incrementó durante la prevalencia de ese régimen, ${ }^{4}$ con el argumento de que el tipo de cambio se ajustaría a las condiciones del mercado y sus fluctuaciones no darían lugar a presiones de aumentos a salarios o precios. El año 1994 estuvo marcado por una serie de acontecimientos sociales, políticos y económicos de naturaleza interna como el levantamiento zapatista en Chiapas, la renuncia del Procurador General de la República y los asesinatos del candidato presidencial Donaldo Colosio y del líder del partido en el poder José Francisco Ruiz Massieu, así como también hechos externos como las alzas en las tasas de interés en Estados Unidos. Estos acontecimientos estuvieron además acompañados de decisiones de política monetaria en virtud de las cuales el peso se encontraba sobrevaluado en términos reales respecto de la moneda estadounidense contribuyendo, desde la perspectiva de estudiosos de los asuntos económicos y cambiarios, a un desplazamiento de la demanda interna hacia los bienes importados. Dornbusch y Werner (1994) son un ejemplo ampliamente conocido de esta posición. Es también sabido que incluso Rudiger Dornbusch sugería públicamente al gobierno mexicano que ajustase la paridad devaluando el peso en $20 \%$ respecto al nivel de cotización de la divisa estadounidense. Calvo (1994) opinaba que una medida de esa naturaleza no sólo no resolvería el desequilibrio existente sino que más bien podría empeorar la situación debido a las expectativas que se podrían generar al implantarla, enfatizando en particular los efectos negativos en el sector financiero dado el deterioro

\footnotetext{
${ }^{4}$ Desde octubre de 1992 el techo de la banda se ajustaba a una tasa de depreciación de 0.0004 nuevos pesos por día, mientras que el piso se mantuvo constante desde esa fecha en 3.0512 nuevos pesos por dólar.
} 
que se produciría en la confianza de los inversionistas. Dada la merma que habían sufrido las reservas internacionales de manera sostenida durante ese año debido a las intervenciones del Banco Central para sostener la paridad cambiaria, es altamente probable que esta última posición contase con fuerte apoyo incluso dentro de las filas de los funcionarios públicos responsables de la conducción de la política económica en el más alto nivel, excepto el Secretario de Hacienda de la época. Con base en su modelos de expectativas autocumplidas, Sachs et al. (1996) afirman que las autoridades mexicanas no devaluaban el peso porque probablemente temían que se perdiera la confianza, pero la pérdida de reservas que se observaba anticipaba que de ocurrir una devaluación ésta sería de magnitud considerable; como consecuencia se generaron condiciones en las cuales podrían presentarse ataques especulativos exitosos.

De una forma u otra, las presiones que se fueron acumulando sobre el tipo de cambio condujeron a que el 20 de diciembre se ajustara la paridad peso-dólar elevando la banda superior de forma que en la práctica el ajuste resultaba ser una abrupta depreciación de 15\%. Se infiere que el ajuste cambiario no fue suficiente para mantener la paridad dentro de las bandas establecidas y así el 22 de diciembre de 1994 la autoridad monetaria mexicana se vio obligada a abandonar sus esfuerzos para sostener el régimen cambiario y finalmente decidió abandonar el tipo de cambio a las fuerzas del mercado, iniciándose de esta forma el régimen de libre flotación de la moneda mexicana que se encuentra vigente hasta la fecha. Como es sabido, una consecuencia del colapso de la moneda mexicana fue que al comenzar 1995 el país se encontraba con una paridad cambiaria por arriba de cinco pesos por dólar e inmerso en un profundo pánico financiero caracterizado por la corrida observada en los mercados de títulos financieros en los cuales se repudiaba, sin distingo alguno, todo tipo de activo, fuese éste emitido por el gobierno o por el sector privado. Para obscurecer aún más el panorama, la economía mexicana entró en una profunda recesión cuyos efectos negativos fueron muy drásticos en el desempeño económico prolongándose hasta mediados de 1996. Es bastante comprensible que las medidas que estableció el gobierno mexicano para enfrentar la crisis desatada no fuesen del agrado 
de las mayorías, generando entre ellas un profundo malestar pues les hizo más onerosa la crisis. Es necesario reconocer que hay quienes opinan que no sólo era el único sino el mejor camino para superarla, argumentado que tales medidas tuvieron como consecuencia el restablecimiento de la confianza así como la estabilidad económica y cambiaria que se vivió después de la recuperación económica. En todo caso resulta evidente que no hay ganadores en el debate sobre la idoneidad de dicha medida.

Podría también atribuirse la estabilidad cambiaria, al menos en parte, al papel que jugaron los derivados en el alivio de las presiones enfrentadas por el mercado de cambios de contado y los agentes económicos al reducir la incertidumbre en torno al comportamiento de la cotización de la divisa estadounidense. Actualmente existe un mercado mexicano de derivados, pero posteriormente al estallido de la crisis el Banco de México autorizó a los bancos, en ciertas condiciones, particularmente las relacionadas con calificación técnica y solvencia, a realizar operaciones en futuros de divisas. En 1995 la autoridad monetaria emitió las disposiciones necesarias para facilitar la operación de contratos sobre el peso mexicano en el mercado de futuros de la Bolsa Mercantil de Chicago.

El Banco Central mexicano realizó diversos esfuerzos con el fin de alcanzar la estabilidad cambiaria. Según Ramos-Francia y Torres (2005), un elemento de la estrategia para restaurar la credibilidad en la política monetaria fue el conjunto de reglas previamente anunciadas mediante las cuales el Banco de México intervino en el mercado cambiario. Uno de los retos a enfrentar era incrementar la acumulación de reservas internacionales para fortalecer la credibilidad en la moneda a fin de aumentar la viabilidad de un régimen cambiario flexible. La principal fuente de reservas internacionales fueron las exportaciones petroleras. Los dólares que se captan por esta vía a través de Petróleos Mexicanos (Pemex), se le venden directamente al Banco de México al tipo de cambio del mercado; según Ramos-Francia y Torres (2005), este mecanismo tiene dos beneficios: permite que el Banco Central acumule reservas internacionales sin intervenciones discrecionales y aísla el mercado cambiario de las variaciones del precio internacional del petróleo. En 1996 la Comisión de Cambios introdujo un instrumento 
explícitamente diseñado para fomentar la acumulación de reservas internacionales, la venta de opciones put una vez al mes para venderle dólares al Banco Central en cualquier día hábil al tipo de cambio interbancario de referencia determinado el día hábil anterior, siempre y cuando ese tipo de cambio no excediera el promedio de los 20 días previos a la fecha de ejercicio. Este instrumento fue diseñado para que los participantes del mercado tuvieran el incentivo de ejercer las opciones cuando el tipo de cambio se apreciara. De esta manera, el Banco de México acumuló reservas sin interferir con el régimen cambiario flotante. En 1997 se introdujo un tercer instrumento para reducir la volatilidad del tipo de cambio en temporadas en las que el mercado cambiario experimentara bajos niveles de liquidez. Esto consistió en un mecanismo automático mediante el cual el Banco Central subastaría 200 millones de dólares en caso de que el tipo de cambio se depreciara, el día que fuese, en más de 2\% respecto del tipo de cambio FIX del día anterior. Ramos-Francia y Torres (2005) sostienen que este instrumento añadió simetría con respecto a la dirección en la que el Banco Central puede intervenir en el mercado cambiario. Esta estrategia para acumular reservas internacionales, que dependía de intervenciones no discrecionales en virtud de que los tres mecanismos seguían reglas previamente anunciadas, permitió que se acumulara una gran cantidad de reservas internacionales a la luz del proceso esperado de remonetización que la economía experimentó como resultado de haberse logrado la estabilización después de la crisis.

De igual forma, con la finalidad de garantizar que la acumulación de reservas internacionales no causara ninguna sobre-expansión de la base monetaria, el Banco de México esterilizó todo impacto sobre la base monetaria más allá del crecimiento de la demanda de dinero.

Benavides y Capistrán (2009) argumentan que las volatilidades de la tasa de interés y del tipo de cambio disminuyeron alrededor de la fecha en que el Banco de México transitó del corto al objetivo de tasas de interés como instrumento de política monetaria. A través de un GARCH bivariado y pruebas de causalidad en varianza, ellos encuentran bicausalidad entre estas variables durante el periodo del corto, pero no encuentran ninguna relación causal después de que empezó la transición. Sin embargo, señalan que hay razones 
para creer que la volatilidad cambiaria debió de haberse incrementado con la introducción de la tasa de interés objetivo. Basándose en Schwartz et al. (2002), Benavides y Capistrán (2009) sostienen que es probable ese incremento en virtud de que los choques externos en una economía pequeña con una política como el corto se capturan tanto por el tipo de cambio nominal como por la tasa de interés nominal, pero con tasas de interés objetivo únicamente el tipo de cambio absorbe esos choques. Un argumento semejante se plantea en Martínez et al. (2001).

En la gráfica 1 se muestra la trayectoria que el tipo de cambio spot peso-dólar ha seguido en su cotización a la venta a partir del dos de enero de 1995 y hasta el doce de agosto de 2010, en total 3928 observaciones. Resulta bastante obvio que la cotización del dólar ha aumentado de manera constante y sostenida, siendo también bastante notorio el salto que el tipo de cambio da a un nuevo nivel entre 2008 y 2009. Naturalmente, ese incremento en el nivel de la cotización del dólar puede verse como un resultado directo de las presiones surgidas por la crisis financiera global cuyo origen se reconoce en el desempeño de la economía estadounidense y el colapso del mercado de hipotecas de ese país. Algo semejante se podría decir de los saltos observados en la cotización del dólar durante 1998 en torno al episodio de la crisis de la deuda rusa.

En la gráfica 2 se muestra la tasa de depreciación (apreciación) diaria de la moneda mexicana respecto del dólar, observada durante el periodo de referencia. Esa tasa también puede considerarse como la de los rendimientos diarios que se pueden tener en términos del peso por la tenencia de dólares. La tasa de depreciación (rendimiento) para cada día se calculó como el logaritmo natural de la razón entre la cotización de ese día y la del anterior, es decir: $r_{t}=\ln \left(y_{t} / y_{t-1}\right)$. Al observar la gráfica 2 se puede suponer apriori que existe una tasa de depreciación (apreciación) diaria del peso cuya variabilidad puede caracterizarse como predominantemente baja a lo largo del horizonte temporal del análisis, observándose únicamente periodos de mayor variabilidad relativamente cortos. Este hecho estilizado brinda apoyo a la decisión de considerar únicamente dos regímenes o estados en el modelo de la volatilidad cambiaria cuya estimación se presenta más adelante. 


\section{GRÁFICA 1}

\section{Cotización del dólar en pesos mexicanos}

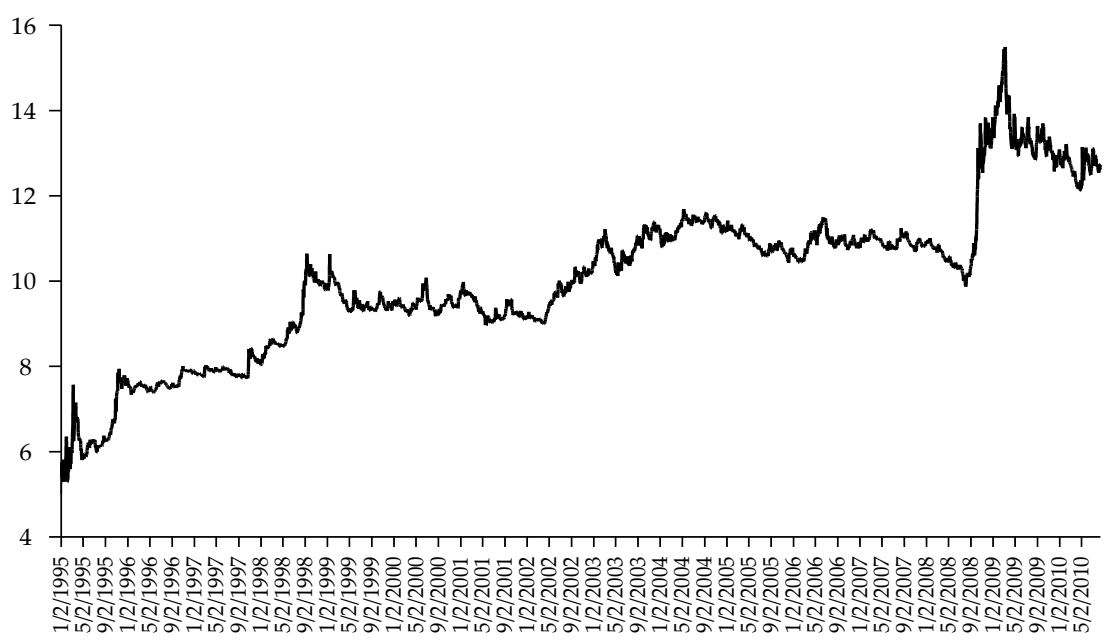

GRÁFICA 2

Tasa de depreciación (rendimiento) del peso frente al dólar

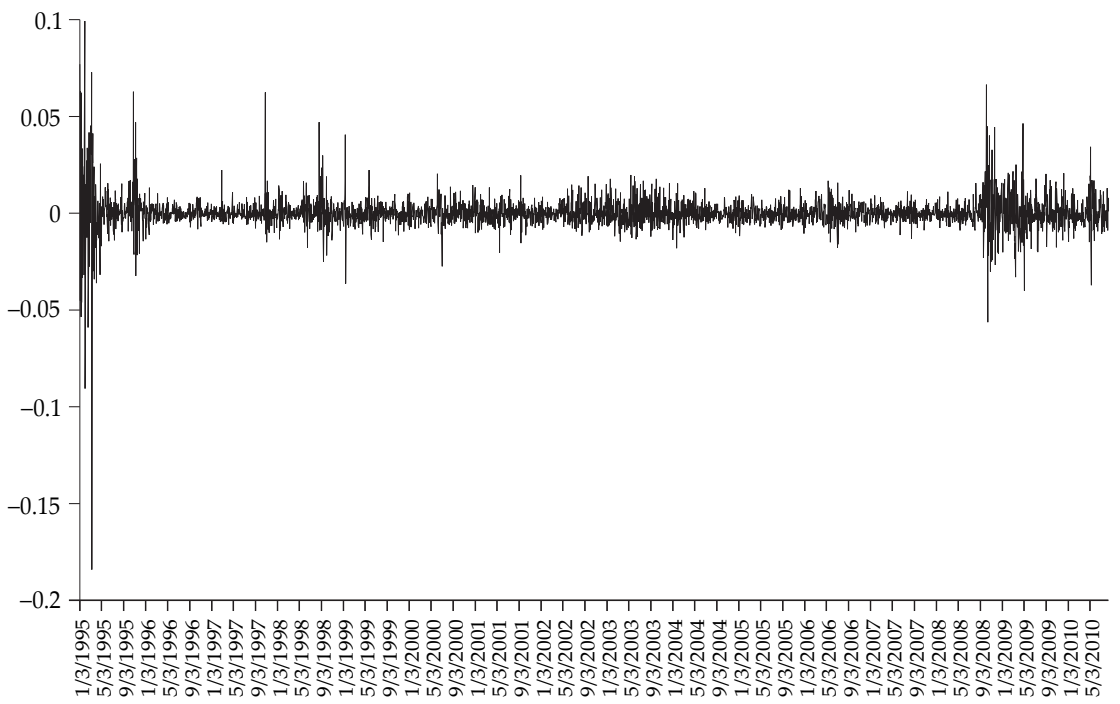


Las principales características estadísticas de la tasa de depreciación del peso mexicano se presentan en el cuadro 1.

\section{Cuadro 1}

Estadísticas descriptivas de la tasa de rendimiento (depreciación) del peso frente al dólar

\begin{tabular}{lc}
\hline Estadístico & Valor \\
\hline Media & 0.000238154 \\
Desviación estándar & 0.008464481 \\
Correlación a un rezago & -0.06826199 \\
Sesgo & -1.724422438 \\
Curtosis & 81.06523628 \\
Mínimo & -0.184177583 \\
Máximo & 0.099254958 \\
\hline
\end{tabular}

La media del rendimiento cambiario (o de la tasa de apreciación/depreciación si se prefiere) es muy baja, lo que es natural al tratarse de datos diarios. No obstante, el coeficiente de variación alcanza un valor considerablemente alto de más de 35, es decir, la desviación estándar (no condicionada) excede más de 35 veces en valor a la media. Se puede decir entonces que la paridad cambiaria del peso mexicano parece ser bastante volátil. Por otra parte, destaca a simple vista la magnitud del exceso de la curtosis, exceso que también es muy común en datos financieros y que en gran parte explica porqué se ha hecho un uso intensivo de modelos de la volatilidad que consideran que ésta es variante en el tiempo. También se observa un ligero sesgo negativo, los movimientos del tipo de cambio se han sesgado hacia el lado de la distribución que puede implicar cierta apreciación de la moneda mexicana o la posibilidad de generar rendimientos positivos por el hecho de contar con moneda nacional en lugar de dólares estadounidenses.

En el cuadro 2 se presentan los resultados de la estimación por el método de máxima verosimilitud del modelo de cadena de Markov para la volatilidad del tipo de cambio peso-dólar que se presentó en la sección previa. En la 
práctica es posible obtener las estimaciones de máxima verosimilitud mediante un algoritmo iterativo que es similar en esencia al algoritmo conocido como Filtro de Kalman. Hamilton $(1989,1994)$ formula de esta manera la solución del problema que implica obtener las estimaciones de variables que no son observables directamente en los datos. Se tomó el valor de la media de la tasa de depreciación (0.000238154) como el valor fijo para $\mu$, opción recomendada por Taylor (2005) pues cabría la posibilidad de que ese parámetro fuese igual a alguno de los valores observados de la tasa de depreciación o rendimiento, caso en el cual se tendría entonces que la función de verosimilitud no converge a ningún valor y no sería posible obtener los valores estimados de los cinco parámetros de interés.

\section{Cuadro 2}

\section{Estimación del modelo}

\begin{tabular}{lc}
\hline Parámetro & Valor estimado \\
\hline$\sigma_{\text {baja }}$ & $0.00429321^{* * *}$ \\
$\sigma_{\text {alta }}$ & $0.02159254^{* * *}$ \\
$p$ & $0.87977054^{* * *}$ \\
$\phi$ & $0.87901679^{* * *}$ \\
Log-verosimilitud & 14831.974642 \\
\hline Nota: $(* *)$ 1\% de significancia. &
\end{tabular}

El valor estimado con nuestro modelo para la volatilidad cambiaria diaria baja es menos de la quinta parte del valor que puede alcanzar la volatilidad alta también en un día. Por otra parte, la probabilidad $(\approx 0.88)$ de que la volatilidad de la cotización del dólar se encuentre en estado de baja volatilidad es bastante alta, razón por la cual se puede afirmar que ese régimen es el prevaleciente en el proceso. Asimismo, con base en los resultados de nuestras estimaciones el periodo esperado para pasar al régimen de alta volatilidad al alcanzar el estado o régimen de baja volatilidad es de alrededor de 68 días, en tanto que se espera que al alcanzar el régimen de alta volatilidad se pase al de baja volatilidad relativamente pronto, en poco más de un día. 
Estos resultados muestran que los dos estados de la volatilidad cambiaria peso-dólar modelados son recurrentes pues al partir de uno de ellos se regresará al mismo en el futuro; lo que es relevante para la administración de riesgos ya que representa una oportunidad de limitar pérdidas derivadas del riesgo cambiario. No obstante, hay que tomar en cuenta que sólo puede estimarse de manera aproximada el tiempo (número de periodos) que tardaría la volatilidad en alcanzar nuevamente el mismo estado una vez que se partió de él y que el agente económico no sabe con certeza en qué estado de volatilidad se encuentra, únicamente se puede inferir la probabilidad de encontrarse en ese estado o régimen.

Se observa también que la persistencia estimada de la volatilidad cambiaria peso-dólar, $\phi \approx 0.879$, no es tan alta en nuestra estimación como lo es de acuerdo con el valor de 0.937 que para ese parámetro estiman Arranz e Iglesias (2005). Este resultado implica que las probabilidades de cambio de régimen de volatilidad deben ser un tanto mayores con el valor de nuestra estimación, haciendo más riesgosa en términos del dólar la posición de un inversionista que mantiene pesos. El valor de nuestra estimación es bastante similar al estimado por Taylor (1999) para el caso del tipo de cambio de la libra esterlina respecto del dólar estadounidense: 0.880 . Incluso cabría destacar al respecto que Arranz e Iglesias (2005) estiman valores incluso inferiores para ese parámetro en los casos de la volatilidad de los tipos de cambio de Noruega y Venezuela, 0.802 y 0.809 respectivamente, y en el caso del Reino Unido el valor que estiman es ligeramente superior: 0.908.

Además, al observar la matriz de probabilidades de transición que se muestra en el cuadro 3, se puede decir que una vez que la volatilidad del tipo de cambio peso-dólar se encuentra en un estado o régimen, es muy baja la probabilidad de que pase al otro estado. Especialmente, se observa que cuando se encuentra en el estado de baja volatilidad es muy poco probable que pase al estado de alta volatilidad, siendo la probabilidad apenas superior a $1 \%$. Es relativamente mayor la probabilidad de que estando la volatilidad en el régimen de volatilidad alta pase al siguiente día al régimen de volatilidad baja, poco más de 10.5 por ciento. 


\section{Cuadro 3}

\section{Matriz de probabilidades de transición}

\begin{tabular}{c|lcc}
\hline & \multicolumn{2}{c}{$\boldsymbol{t}$} \\
\cline { 2 - 4 } \multicolumn{2}{c}{} & $\sigma_{\text {baja }}$ & $\sigma_{\text {alta }}$ \\
\hline \multirow{2}{*}{$t-1$} & $\sigma_{\text {baja }}$ & 0.98545425 & 0.014545746 \\
& $\sigma_{\text {alta }}$ & 0.10643747 & 0.893562535 \\
\hline
\end{tabular}

En la gráfica 3 se observan las varianzas condicionales y las probabilidades, también condicionales, de que la volatilidad se encuentre en el estado o régimen de baja volatilidad. Se observa que el año 1995 estuvo marcado por un alto nivel de volatilidad cambiaria; como es sabido, durante ese periodo el peso sufrió importantes depreciaciones respecto del dólar, en particular durante los primeros meses del año.

\section{GRÁFICA 3 \\ Varianzas condicionales y probabilidades condicionales del régimen de baja volatilidad}

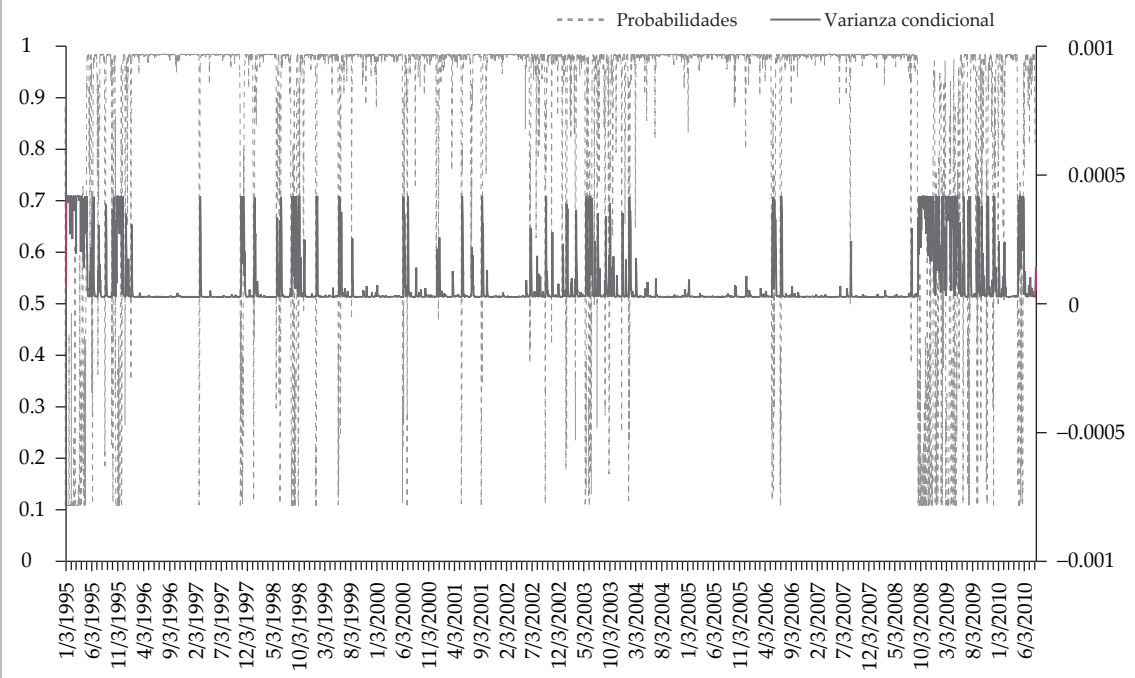


En la misma gráfica 3 se puede ver que durante ese periodo las probabilidades condicionadas de encontrarse en el régimen de baja volatilidad eran muy bajas, alternativamente podríamos decir que eran altas las probabilidades de encontrarse en el régimen de alta volatilidad. De acuerdo con informes del Banco de México, después de observarse un periodo de relativa calma, en octubre de 1995 hay otro episodio de alta volatilidad del tipo de cambio. En nuestro modelo se captura el alza de la volatilidad en ese mes pero antes se observa que estuvo precedido de algunos saltos de volatilidad, posteriormente se van incrementando las probabilidades condicionales de baja volatilidad, manteniéndose así durante prácticamente todo 1996. A pesar de que durante octubre y noviembre de 1996 se observa un ascenso sostenido en el tipo de cambio, debido a lo cual se sitúa alrededor de ocho pesos por dólar, en el modelo no se observa que la volatilidad cambiaria haya sufrido un alza importante, resultado congruente con lo reportado por la autoridad monetaria mexicana.

Otros periodos conocidos de alta volatilidad cambiaria que se reconocen en el modelo es el que inicia con la crisis asiática a partir de julio de 1997, después de que en febrero de ese mismo año se observó un periodo de alza en la volatilidad. Algo semejante podemos decir de los periodos asociados con la crisis rusa de agosto de 1998 y la crisis brasileña de enero de 1999. En el primer caso observamos que el periodo de alta volatilidad se prolonga hasta noviembre de 1998, en tanto que en el segundo caso la volatilidad regresa muy rápidamente a un nivel bajo. Entre 2000 y 2001 se encuentran periodos relativamente cortos caracterizados por alzas en la volatilidad cambiaria alternándose con periodos de baja volatilidad que también son relativamente cortos. Parece razonable atribuir los episodios de alta volatilidad del año 2001, al menos en parte, a la desaceleración de la economía de Estados Unidos, la cual produjo caídas en las exportaciones, la producción, el empleo y el precio de la mezcla mexicana del petróleo, generando incertidumbre respecto al desempeño económico y expectativas de depreciación cambiaria. 
Después de que durante 2006 y 2007 se observase predominantemente baja volatilidad, ${ }^{5}$ de agosto de 2008 a mayo de 2010 se observan periodos de alta volatilidad muy cercanos entre sí, únicamente interrumpidos por periodos de baja volatilidad con duración relativamente corta. La explicación que se puede dar a esos periodos es la conjugación de factores tales como la crisis financiera global disparada por la crisis de las hipotecas subprime y sus efectos en los mercados financieros mundiales, la recesión observada primero en Estados Unidos y después a escala mundial, los temores sobre los efectos globales de la crisis griega, disparada durante el primer semestre de 2010, y finalmente la consiguiente incertidumbre respecto del comportamiento de la economía nacional en el futuro inmediato. De manera general, podemos decir que, según nuestro modelo, en la volatilidad del tipo de cambio pesodólar se observan periodos más o menos prolongados de baja volatilidad, interrumpidos por periodos en los cuales la volatilidad es alta.

\section{Conclusiones}

En este trabajo se ha analizado la volatilidad cambiaria peso-dólar mediante un modelo de volatilidad estocástica con un enfoque markoviano. Este enfoque ha permitido identificar y caracterizar los periodos de baja y alta volatilidad mediante la estimación de las probabilidades de ocurrencia asociadas a cada uno de ellos.

El sesgo en favor del fortalecimiento del peso frente a la moneda de Estados Unidos se ha derivado, podría suponerse, del comportamiento de algunos de los fundamentales económicos como lo argumenta la autoridad monetaria mexicana. En particular, las políticas fiscales y monetarias altamente restrictivas que fueron instrumentadas después de la crisis de 1995, así como los programas afines aplicados para reestructurar el sistema financiero mexicano, parecen haber contribuido a que se observe en el tipo de cambio

\footnotetext{
${ }^{5}$ Durante algunos días del mes de agosto de 2007 se observaron altas volatilidades, muy probablemente asociadas con el inicio de las dificultades en el mercado de hipotecas de Estados Unidos y sus efectos en los mercados financieros del mundo.
} 
peso-dólar un comportamiento relativamente ordenado, caracterizado por periodos prolongados de calma relativa con baja volatilidad, alternándose con algunos periodos de alta volatilidad.

Quizá mediante el análisis de las intervenciones del Banco de México en el mercado cambiario, en el marco del régimen de flotación administrada, se podría explicar el que Bazdresch y Werner (2002) hayan encontrado que existe un sesgo en los mercados de forwards según el cual se pronostican depreciaciones del peso que son mayores a las observadas. Es decir, en su análisis Bazdresch y Werner detectan la presencia de una variante del denominado peso-problem, término cuya acuñación es atribuida a Milton Friedman para describir la estabilidad del peso mexicano a principios de los años setenta del siglo pasado a pesar de que el diferencial entre las tasas de interés sugería que las autoridades mexicanas debían llevar a cabo una devaluación del peso mexicano para corregir el desajuste. Con base en su modelo de cambio de régimen, Bazdresch y Werner concluyen que la variante que encuentran del peso-problem se explica en parte como consecuencia de la probabilidad latente de pasar a un régimen de alta volatilidad con depreciaciones pronunciadas a pesar de que no se haya observado ese régimen incluso durante un periodo prolongado. Nuestros resultados son congruentes con lo planteado por Bazdresch y Werner, sugiriendo que el peso-problem subsiste aún a pesar del régimen de libre flotación y de la estabilidad macroeconómica.

A pesar de que la volatilidad cambiaria de la moneda mexicana ha mostrado una estabilidad relativa y de que el tipo de cambio ha mantenido una tendencia alcista desde que se optó por el régimen de libre flotación, algunos analistas sostienen que la moneda nacional está sobrevaluada respecto del nivel que corresponde al tipo de cambio de equilibrio. En la medida en que se generalizase esta percepción habría expectativas de una depreciación del peso mexicano y, en términos del mercado cambiario, se acumularían presiones para que ocurriese la depreciación como factor correctivo del desajuste cambiario incluso aunque las probabilidades de ese movimiento correctivo fuesen relativamente bajas (pequeñas inclusive). Una consecuencia importante de la probabilidad latente de observar alta volatilidad del tipo de cambio y con ella cambios bruscos en la paridad peso-dólar, es la necesidad 
de que los agentes económicos, en particular las empresas e inversionistas de portafolio, cubran sus exposiciones al riesgo cambiario. Al respecto, para diseñar y valuar tales coberturas es importante que se consideren las características del proceso de la volatilidad cambiaria, la cual es variante en el tiempo como se mostró en páginas previas y que además puede encontrarse en diferentes estados o regímenes.

Por otra parte, es importante considerar que durante el periodo de estudio las intervenciones del Banco de México en el mercado cambiario no parecen haber sido siempre eficaces para reducir la volatilidad cambiaria. Como ejemplo se pueden mencionar las intervenciones durante el periodo de crisis 2008-2009, cuando hubo importantes inyecciones de dólares por parte de la autoridad monetaria para estabilizar el tipo de cambio pero el impacto sobre la volatilidad cambiaria fue casi siempre inocuo. Es decir, tales intervenciones no sólo fueron costosas en términos de la pérdida inherente de reservas internacionales sino que tampoco redujeron el riesgo cambiario ni la necesidad de coberturas y los costos asociados con éstas. Evidentemente esto pone en evidencia que las intervenciones del Banco Central como política de estabilización cambiaria son limitadas e incluso podrían ser subóptimas. Esto no quiere decir que la autoridad monetaria deba abandonar la intervención directa en el mercado de cambios, lo que se pone de manifiesto es la necesidad de que se desarrollen políticas e instrumentos más eficaces para acompañar su intervención reduciendo el riesgo cambiario. Tales políticas e instrumentos deben incluir objetivos acordes con el desarrollo económico del país, al mismo tiempo que toman en consideración las expectativas de los agentes económicos.

La evidencia que aquí se ha mostrado sugiere que las acciones del Banco de México han sido eficaces para la estabilización cambiaria, pero para una evaluación más objetiva de la actuación del Banco Central mexicano se deben considerar los costos del sostenimiento de una paridad relativamente estable, en la cual juegan un papel importante las políticas restrictivas de contención de las presiones inflacionarias que no fomentan el crecimiento económico. De acuerdo con Galindo y Ros (2008), los efectos de las variaciones cambiarias sobre la inflación (pass-through) han disminuido pero no se han eliminado del 
todo, lo que implica que para alcanzar los objetivos de inflación de acuerdo con el esquema establecido por el Banco de México, éste tenga que hacer esfuerzos para lograr la estabilidad cambiaria. El problema radica en que la estabilidad en el nivel de los precios internos puede estar significando un costo real en términos de sacrificio del empleo y el producto, como lo argumenta Perrotini (2007), lo que evidentemente puede representar en última instancia pérdida de oportunidades de desarrollo que redunden en mejorías notables en el nivel de vida de toda la población del país.

\section{REFERENCIAS}

Arranz, M. y E.M. Iglesias, "Análisis de los tipos de cambio en la economía mexicana y comparación con otros países: un enfoque de volatilidad estocástica", Investigación Económica, vol. 64, núm. 253, 2005, pp. 159-169.

Baum, L.E.; T. Petrie; G. Soules y N. Weiss, "A maximization technique occurring in the statistical analysis of probabilistic functions of Markov chains", Annals of Mathematical Statistics, núm. 41, 1980, pp. 164-171.

Baillie, R.T.; T. Bollerslev y H.O. Mikkelsen, "Fractionally integrated generalized autoregressive conditional heteroskedasticity", Journal of Econometrics, núm. 74, 1996, pp. 3-30.

Baum, Ch.F. y M. Caglayan, "The volatility of international trade flows and exchange rate uncertainty", Department of Economics, Boston College y Department of Economics, University of Sheffield, Working paper, 2009.

Bauwens, L. y G. Sucarrat, "General to specific modelling of exchange rate volatility: a forecast evaluation”, Departamento de Economía, Universidad Carlos III de Madrid, Working Paper 08-18 Economic Series (10), 2008.

Bazdresch, C. y A. Werner, "El comportamiento del tipo de cambio en México y el régimen de libre flotación 1996-2001”, Banco de México, Documento de Investigación no. 2002-09, 2002.

Benavides, G. y C. Capistrán, "Una nota sobre las volatilidades de la tasa de interés y del tipo de cambio según diferentes instrumentos de política monetaria: México, 1998-2008”, Banco de México, Documento de Investigación no. 2009-10, 2009.

Black, F. y M. Scholes, "The valuation of option contracts and a test of market efficiency”, Journal of Finance, núm. 27, 1972, pp. 399-418. 
Calvo, G., "Comment on Dornbusch and Werner", Brookings Papers on Economic Activity, 1994, pp. 253-315.

Chowdhury, I. y L. Sarno, "Time-varying volatility in the foreign exchange market: new evidence on its persistence and on currency spillovers", Journal of Business Finance and Accounting, núm. 31, 2004, pp. 759- 793.

Clark, P.B., "Uncertainty, exchange risk, and the level of international trade", Western Economic Journal, núm. 11, 1973, pp. 302-313.

Clark, P.B.; N. Tamirisa; S. Wei; A. Sadikov y L. Zeng, "Exchange rate volatility and trade flows-Some new evidence", International Monetary Fund (IMF), Working Paper no. 051904, 2004.

Cushman, D.O., "U.S. bilateral trade flows and exchange risk during the floating period", Journal of International Economics, núm. 25, 1988, pp. 317-330.

De Grauwe, P., "Exchange rate variability and the slowdown in the growth of international trade", IMF, Staff Papers no. 35, 1988, pp. 63-84.

Dornbusch, R. y A. Werner, "Mexico: stabilization, reform, and no growth", Brookings Papers on Economic Activity, 1994, pp. 253-315.

Engel, C. y J.D. Hamilton, "Long swings in the dollar: are they in the data and do markets know it?", American Economic Review, núm. 80, 1990, pp. 689-713.

Ethier, W., "International trade and the forward exchange market", American Economic Review, núm. 63, 1973, pp. 494-503.

Fama, E.F., “The behavior of stock market prices", Journal of Business, núm. 38, 1965, pp. 34-105.

Franke, G., "Exchange rate volatility and international trade strategy", Journal of International Money and Finance, núm. 10, 1991, pp. 269-287.

Galindo, L.M. y J. Ros, "Alternatives to inflation targeting in Mexico", International Review of Applied Economics, vol. 22, núm. 2, 2008, pp. 201-214.

Giovannini, A., "Exchange Rate and Traded Goods Prices", Journal of International Economics, núm. 24, 1988, pp. 45-68.

Goldfeld, S.M. y R.E. Quandt, "A Markov model for switching regressions”, Journal of Econometrics, núm. 1, 1973, pp. 3-16.

Hamilton, J.D., "Rational-expectations econometric analysis of changes in regime: an investigation of the term structure of interest rates", Journal of Economic Dynamics and Control, núm. 12, 1988, pp. 385-423.

, "A new approach to the economic analysis of nonstationary time series and the business cycle", Econometrica, núm. 57, 1989, pp. 357-384.

—, Time Series Analysis, Princeton, N.J., Princeton University Press, 1994, pp. 799. 
, "What's real about the business cycle?", Federal Reserve Bank of St. Louis Review, vol. 87, núm. 4, 2005, pp. 435-452.

Hooper, P. y S.W. Kohlhagen, "The effects of exchange rate uncertainty on the prices and volume of international trade", Journal of International Economics, núm. 8, 1978, pp. 483-511.

Jeanne, O. y P. Masson, "Currency crisis, sunspots and Markov-switching regimes", Journal of International Economics, vol. 50, núm. 2, 2000, pp. 327-350.

Kellard, N.; P. Newbold y T. Rayner, "Evaluating currency market efficiency: are cointegration tests appropriate?”, Applied Financial Economics, núm. 11, 2001, pp. 681-691.

López-Herrera, F. y E. Ortiz, "Dynamic multibeta macroeconomic asset pricing model at NAFTA stock markets", International Journal of Economics and Finance, vol. 3, núm. 1, 2011, pp. 55-68.

Lindgren, G., "Markov regime models for mixed distributions and switching regressions", Scandinavian Journal of Statistics, núm. 5, 1978, pp. 81-91.

Longmore, R. y W. Robinson, "Modelling and forecasting exchange rate dynamics: an application of asymmetric volatility models", Research Services Department, Bank of Jamaica, Working Paper no. WP2004/03, 2004.

Mahieu, R.J. y P.C. Schotman, "An empirical application of stochastic volatility models", Journal of Applied Econometrics, vol. 13, núm. 4, 1998, pp. 333-360.

Mandelbrot, B., "The variation of certain speculative prices", Journal of Business, núm. 36, 1963, pp. 394-419.

Martínez, L.; O. Sánchez y A. Werner, "Consideraciones sobre la conducción de la política monetaria y el mecanismo de transmisión en México", Banco de México, Documento de Investigación no. 2001-02, 2001.

Masson, P., "Exchange rate regimes transition”, IMF, Working Paper no. 00-134, 2000. McMillan, D.G. y A.E.H. Speight, "Long memory and heterogeneous components in high frequency pacific-basin exchange rate volatility", Asia-Pacific Financial Markets, núm. 12, 2006, pp. 199-226.

McQueen, G.Y. y S. Thorley, “Are stock returns predictable? A test using Markov chains”, Journal of Finance, vol. 46, núm. 1, 1991, pp. 239-263.

Mohnot, R., "Forecasting Forex volatility in turbulent times", Global Journal of Business Research, vol. 5, núm. 1, 2011, pp. 27-38.

Neftci, S., "Are economic time series asymmetric over the business cycle?", Journal of Political Economy, núm. 92, 1984, pp. 307-328. 
Olowe, R.A., "Modelling naira/dollar exchange rate volatility: application of GARCH and assymetric models", International Review of Business Research Papers, vol. 5, núm. 3, 2009, pp. 377-398.

Pagan, A.R., "The econometrics of financial markets", Journal of Empirical Finance, vol. 3, núm. 1, 1996 , pp.15-102.

Perrotini, I., "El nuevo paradigma monetario", Economía UNAM, vol. 4, núm. 11, 2007, pp. 64-82.

Perrotini, I. y D. Rodríguez, "Assessing the efficiency of the forward exchange rate market of the Mexican peso, 1995-2003”, en I. Perrotini Hernández y F. Zaher (eds.), Studies of Sweden and Mexico. Economics, Finance, Trade and Environment, Sweden, University of Skövde, 2007.

Prasad, N.R.; R.C. Ender; S.T. Reilly y G. Nesgos, "Allocation of resources on a minimized cost basis", 1974 IEEE Conference on Decision and Control including the 13th Symposium on Adaptive Processes 13, 1974 , pp. 402-403.

Ramírez, J.C. y R. Sandoval-Saveedra, “¿Existen componentes pronosticables en las series de los rendimientos de las acciones?”, Revista Mexicana de Economía y Finanz̧as, núm. 11, 2002, pp. 39-58.

Ramos-Francia, M. y A. Torres, "Reducing inflation through inflation targeting: the Mexican experience", Banco de México, Documento de Investigación no. 2005-01, 2005.

Rodríguez, D. y F. Venegas-Martínez, "La restricción externa al crecimiento en México: 1988-2009", Contaduría y Administración, en prensa.

Sachs, J.; A. Tornell y A. Velasco, "The Mexican peso crisis: sudden death or death foretold?, Journal of International Economics, vol. 41, núms. 3-4, 1996, pp. 265-283.

Sandoval, J., "Do asymmetric GARCH models fit better exchange rate volatilities on emerging markets?”, Odeon, núm. 3, 2006, pp. 97-118.

Sengupta, J.K. y R.E. Sfeir, "Modelling exchange rate volatility", Department of Economics, University of California en Santa Barbara y School of Business, Chapman University, Working paper, 1996.

Siddiqui, M.A., "Modelling pak rupee volatility against five major currencies in the perspective of different exchange rate regimes", European Journal of Economics, Finance and Administrative Sciences, núm. 17, 2009, pp. 81-96.

Schwartz, M.J.; A. Tijerina y L. Torre, "Volatilidad del tipo de cambio y tasas de interés en México: 1996.2001”, Economía Mexicana, vol. XI, núm. 2, 2002, pp. 299-331. 
Taylor, S.J., "Financial returns modelled by the product of two stochastic processes: a study of the daily sugar prices 1961-1975", en O.D. Anderson (ed.), Time Series Analysis: Theory and Practice, vol. 1, Amsterdam, North-Holland, 1982, pp. 203-226.

— Modelling Financial Time Series, Chichester, John Wiley, 1986.

- "Modelling stochastic volatility: a review and comparative study", Mathematical Finance, vol. 4, núm. 2, 1994, pp. 183-204.

— "Markov processes and the distribution of volatility: a comparison of discrete and continuous specifications", Philosophical Transactions: Mathematical, Physical and Engineering Sciences, vol. 357, núm. 1758, 1999, pp. 2059-2070.

—., Asset price dynamics, volatility, and prediction, Princeton, N.J., Princeton University Press, 2005.

Tse, Y.K., "The conditional heteroscedasticity of the yen-dollar exchange rate", Journal of Applied Econometrics, vol. 13, núm. 1, 1998, pp. 49-55.

Wold, H.O., A study in the analysis of stationary time series, Uppsala, Almquist and Wicksell, 1938.

Wolf, A., "Import and hedging uncertainty in international trade", Journal of Future Markets, núm. 15, 1995, pp. 101-110. 